\title{
Antibiotics for Upper Respiratory Tract Infections in Ambulatory Practice in the United States, 1997-1999: Does Physician Specialty Matter?
}

\author{
Olivier T. Rutschmann, MD, MPH, and Marisa Elena Domino, PhD
}

Background: The dangers of overuse of antibiotics for upper respiratory infections (URIs) has been widely recognized, but the rate of change in prescribing patterns in recent years is unknown.

Methods: Data on the use of antibiotics for URIs was extracted from the 1997 to 1999 National Ambulatory Medical Care Survey (a national multistage probability sample survey of patients' office visits to office-based physicians). Adult patients ( $\geq 18$ years) with a primary diagnosis of URI (bronchitis, common colds, and other acute upper respiratory infections) were included. The decision to prescribe antibiotics was modeled as a function of patient, physician, and practice characteristics using logistic regression.

Results: The rate of antibiotic prescription for URIs decreased from $52.1 \%$ in 1997 to $41.5 \%$ in 1999. In a multivariate logistic regression model, treatment by general internal medicine physicians [odds ratio (OR), 0.37 ; $95 \%$ confidence interval $(\mathrm{CI}), 0.18$ to 0.76 ] was associated with lower prescription rates. Of patients visiting general internal medicine physicians for URIs, 36.2\% received antibiotics compared with $\mathbf{4 2 . 9 \%}$ of those seeing a general/family medicine physician. Patients treated by their primary care physicians had a higher risk of receiving antibiotics (OR, 1.70; 95\% CI, 1.08 to 2.68).

Conclusions: Despite a downward trend in antibiotic prescribing over the years, overprescription of antibiotics for upper respiratory infections persists. General internal medicine physicians are less likely than general/family physicians to prescribe antibiotics, but this gap seems to be narrowing. Specific interventions must be designed to address these disparities. (J Am Board Fam Pract 2004;17:196-200.)

Overuse of antibiotics in adult ambulatory practice is one of the contributing factors to the increase of antibiotic-resistant bacteria in the community. ${ }^{1}$ In particular, penicillin resistance in Streptococcus pneumoniae has increased dramatically during the last 10 years and previous antibiotic use has been identified as a major risk factor for carriage and spread of antibiotic-resistant $S$ pneumoniae. ${ }^{2}$

Although an overwhelming majority of upper respiratory tract infections are of viral origin, ${ }^{3}$ more than $50 \%$ of patients diagnosed with colds, upper respiratory tract infections, or bronchitis were prescribed antibiotics in an analysis of the

Submitted, revised, February 12, 2004.

From the Duke Center for Clinical Health Policy Research, Duke University Medical Center, Durham, North Carolina and the Department of Health Policy and Administration, School of Public Health, University of North Carolina, Chapel Hill. Address correspondence to Olivier T. Rutschmann, Department of Medicine, Division of Emergency Medicine, Geneva University Hospital, 24 rue Micheli-du-Crest, 1211 Geneva 14, Switzerland (e-mail: olivier.rutschmann@hcuge.ch).

This work was supported in part by a grant from the Swiss Academy of Medical Sciences (to OTR).
1992 National Ambulatory Medical Care Survey (NAMCS). ${ }^{4}$ Despite an increasing awareness of the problem, there is little evidence that practice has changed in recent years.

The objective of this study is to explore whether antibiotic prescription practices for upper respiratory tract infections have changed and to determine the influence of physician and practice characteristics on antibiotic prescribing.

\section{Methods}

To identify the influence of patient and physician characteristics on antibiotic prescribing for URIs, we build on earlier work conducted by Gonzales and colleagues ${ }^{4}$ using an earlier version of the NAMCS. Our study expands on Gonzales and colleagues by providing more recent estimates and investigating time trends in prescribing rates by physician specialty for 1997 to 1999 .

\section{Source of Data}

Data were extracted from the 1997 to 1999 NAMCS datasets. The NAMCS is a national multistage probability sample survey of patients' 
office visits to office-based physicians conducted annually by the Division of Health Care Statistics, National Center for Health Statistics, Centers for Disease Control and Prevention. ${ }^{5}$ The survey includes data on physician, patient, and visit characteristics. All characteristics in the NAMCS are reported by the physician or practice and should be interpreted as such. Antibiotic prescribing as discussed in this article refers to prescriptions written, because the survey does not track prescriptions filled.

\section{Patient Selection}

Our analyses were restricted to visits by adults $(\geq 18$ years). Patients with chronic lung diseases, congestive heart failure, and diabetes (ICD-9-CM codes 491 to $493,250,428$ ) were excluded from the analyses, because broader indications for antibiotics might exist for such patients.

\section{Identification of Predictors for Antibiotic Prescription}

We model the decision to prescribe antibiotics for a URI as a function of patient, physician, and practice characteristics using logistic regression.

Antibiotics coded as primary medication were identified using the "National Drug Code Directory" drug class "antimicrobial agents." For this analysis, only patients with a primary diagnosis of URIs as recorded by the physician were considered; URIs were defined as a group of diseases including bronchitis, common colds, and other acute upper respiratory infections, identified by ICD-9-CM codes (461, 473, 466, 490, 464.10, 464.2, 460, 465). Patients who had a secondary or tertiary diagnosis of sinusitis (codes 461, 473), pneumonia (481 to $483,485$ to 486$)$, urinary tract infection $(590,595$, 599), pharyngitis $(462,463)$, otitis media (381.0, $381.3,381.4,382.0,382.9)$, or skin infections (680 to $684,685.0,686.9,695.3,704.8,706.1$ to 2 ) were excluded, because these diagnoses might be indications for antibiotic prescription.

Predictors of antibiotic prescription used in the model included patient characteristics, practice characteristics, physician characteristics, insurance characteristics, and visit characteristics (Table 1). These variables were used to calculate adjusted odds ratios.

Physicians were stratified into 3 groups: general internal medicine, general/family medicine, and other specialists (including pediatricians, surgeons and all other specialists). In addition, we control for whether a physician reports him or herself as a primary care physician for the specific patient reported in the analyses. Time trends in prescribing rates were explored using indicator variables for each of the survey years; year variables were interacted with (ie, multiplied by) specialty training type to determine time trends for each type of physician. Observations were weighted to the population level and standard errors were adjusted for clustering at the physician level because of the multiple observations contributed by some physicians.

\section{Results}

A total of 141,612,037 antibiotic prescriptions were written between 1997 and 1999. Upper respiratory infections, as recorded by the physicians, were the leading reason for antibiotic prescription $(17.7 \%$ of the total antibiotic prescriptions). Antibiotics were prescribed in $47.8 \%$ of URIs ( $54.3 \%$ of bronchitis and $41.5 \%$ of common colds). Between 1997 and 1999, the prescription rates for URIs decreased from $52.1 \%$ in 1997 to $49.1 \%$ in 1998 and $41.5 \%$ in 1999.

Significant differences in antibiotic prescribing rates were observed across physician training types and primary care provider status. General internal medicine physicians were substantially less likely to prescribe antibiotics for URIs than were general/ family medicine physicians, with an estimated odds ratio of 0.37 . Other types of specialists demonstrated no significant differences from rates of prescribing by general/family medicine physicians, controlling for all other model characteristics. In addition, a $70 \%$ increase in the odds of being prescribed antibiotics was observed if the physician was the primary care physician of the patient.

Predictors of antibiotic prescription were explored for the 956 office visits for URIs that met inclusion and exclusion criteria. Adjusted odds ratios for the different predictors used in our logistic regression model are presented in Table 1 . Personal characteristics, such as age, gender, and race, were not differentially associated with antibiotic prescribing; only Hispanic ethnicity was significantly associated with a marked decline in antibiotic prescribing over that observed for nonHispanics. This effect was not observed in the earlier analysis by Gonzales and colleagues. ${ }^{4}$ In addition, insurance and practice characteristics 
Table 1. Possible Predictors of Ambulatory Antibiotic Prescription for Upper Respiratory Infections in the United States, 1997 to 1999

\begin{tabular}{|c|c|c|}
\hline Predictors & $\begin{array}{l}\text { Office Visit Sample } \\
\text { for a URI }[\%(n)]\end{array}$ & $\begin{array}{l}\text { Adjusted Odds Ratios* } \\
\text { ( } 95 \% \text { CI) for } \\
\text { Antibiotic Prescription }\end{array}$ \\
\hline \multicolumn{3}{|l|}{ Age } \\
\hline $18-44$ & $47 \%(449)$ & Referent \\
\hline $45-64$ & $30 \%(287)$ & $0.96(0.68$ to 1.36$)$ \\
\hline$\geq 65$ & $23 \%(220)$ & $0.80(0.46$ to 1.40$)$ \\
\hline Female vs male & $62 \%(593)$ & $1.03(0.73$ to 1.44$)$ \\
\hline Nonwhite race vs white & $13 \%(124)$ & $0.83(0.50$ to 1.36$)$ \\
\hline Hispanic ethnicity vs not Hispanic & $7 \%(67)$ & 0.17 (0.08 to 0.38$)$ \\
\hline \multicolumn{3}{|l|}{ Geographic region } \\
\hline South & $36 \%(343)$ & Referent \\
\hline Midwest & $26 \%(249)$ & $1.43(0.85$ to 2.41$)$ \\
\hline Northeast & $19 \%(182)$ & $1.33(0.76$ to 2.32$)$ \\
\hline West & $19 \%(182)$ & $1.24(0.72$ to 2.11$)$ \\
\hline Rural area vs urban & $22 \%(210)$ & $1.28(0.80$ to 2.05$)$ \\
\hline \multicolumn{3}{|l|}{ Reimbursement type } \\
\hline Private insurance & $58 \%(555)$ & Referent \\
\hline Medicare & $17 \%(163)$ & $1.11(0.58$ to 2.12$)$ \\
\hline Medicaid & $8 \%(76)$ & $1.09(0.54$ to 2.20$)$ \\
\hline Selfpay & $9 \%(86)$ & $1.30(0.72$ to 2.36$)$ \\
\hline Other & $8 \%(76)$ & $1.17(0.64$ to 2.15$)$ \\
\hline HMO vs not HMO & $27 \%(258)$ & $0.94(0.62$ to 1.43$)$ \\
\hline \multicolumn{3}{|l|}{ Physician specialty } \\
\hline General/family medicine & $53 \%(508)$ & Referent \\
\hline General internal medicine & $36 \%(343)$ & $0.37(0.18$ to 0.76$)$ \\
\hline Other specialties & $11 \%(105)$ & $0.78(0.33$ to 1.86$)$ \\
\hline $\begin{array}{l}\text { Primary care physician for this patient vs not } \\
\text { primary care physician }\end{array}$ & $81 \%(774)$ & 1.70 (1.08 to 2.68$)$ \\
\hline Solo practice vs not solo practice & $40 \%(382)$ & $1.10(0.76$ to 1.61$)$ \\
\hline \multicolumn{3}{|l|}{ Visit duration } \\
\hline$\leq 10$ minutes & $36 \%(344)$ & Referent \\
\hline $10-15$ minutes & $40 \%(382)$ & $0.99(0.65$ to 1.50$)$ \\
\hline$>15$ minutes & $24 \%(230)$ & $1.08(0.69$ to 1.69$)$ \\
\hline $\mathrm{X}$-ray during visit vs not $\mathrm{X}$-ray & $9 \%(86)$ & $1.06(0.64$ to 1.74$)$ \\
\hline \multicolumn{3}{|l|}{ Year of survey } \\
\hline 1997 & $30 \%(287)$ & Referent \\
\hline 1998 & $40 \%(382)$ & $0.64(0.36$ to 1.15$)$ \\
\hline 1999 & $30 \%(287)$ & $0.50(0.27$ to 0.94$)$ \\
\hline \multicolumn{3}{|l|}{ Interaction terms: } \\
\hline General internal medicine * year 1998 & & $2.22(0.92$ to 5.35$)$ \\
\hline General internal medicine * year 1999 & & $2.02(0.45$ to 4.65$)$ \\
\hline Other specialties * year 1998 & & $1.44(0.45$ to 4.65$)$ \\
\hline Other specialties * year 1999 & & $1.51(0.45$ to 5.16$)$ \\
\hline
\end{tabular}

* Adjusted odds ratios are reported from a logistic regression analysis using all variables reported in Table 1.

were not significantly associated with antibiotic prescribing during the sample period. It was surprising that participation in an HMO-type plan (as reported by the physician) did not serve as a protective factor against antibiotic prescribing, despite the potential for provider profiling or other types of utilization review often present in HMO-type plans.

\section{Time Trends in Prescribing}

To determine time trends in antibiotic prescription, we calculated predicted rates of prescription for a standard patient. To be representative, a standard patient was defined as the most commonly observed patient in the data set (ie, white woman consulting her primary care physician for an URI). Predicted rates of prescription for this standard patient, reported in Table 2, show a decrease in prescription rates for general/family medicine physicians during the 3 years of observation. For general internal medicine physicians, an increase in the

Table 2. Predicted Rates (\%) of Antibiotic Prescribing for Patients Consulting Their Primary Care Physician for URIs, by Year and Physician Specialty

\begin{tabular}{llll}
\hline Specialty & 1997 & 1998 & 1999 \\
\hline General/Family Medicine & $59.9 \%$ & $49 \%$ & $42.9 \%$ \\
General Internal Medicine & $35.8 \%$ & $44.3 \%$ & $36.2 \%$ \\
Other Specialties & $53.9 \%$ & $52.1 \%$ & $47.1 \%$ \\
\hline
\end{tabular}


prescription rate was observed between 1997 and 1998, but in 1999 this rate returned to a rate similar to that observed in 1997. The decrease in the rate of prescribing for other specialists was not statistically significant.

\section{Discussion}

Despite overwhelming evidence that overuse of antibiotics is detrimental, that most URIs are cured without treatment, and therefore that the lowest antibiotic prescription rates should be desirable, antibiotics were still prescribed for more than $40 \%$ of the URIs seen in adult ambulatory practice between 1997 and 1999 in the United States. These rates compare favorably with rates observed in older NAMCS datasets ${ }^{4,6}$ or in other populations $(51 \%$ to $85 \%$ of patients prescribed with antibiotics), ${ }^{7}$ and are similar to that observed by Stone and colleagues ${ }^{8}$ in 1996 in emergency departments. A decrease in antibiotic prescriptions for URIs was observed during the 3 years of our analysis, suggesting that awareness about the misuse of antibiotics has increased during these years.

Looking at predictors for antibiotic prescription, general internal medicine physicians had a $63 \%$ lower risk of prescribing antibiotics for URIs than general/family medicine physicians in 1997. However, by 1999, this gap had narrowed, entirely because of changes in rates by general/family medicine physicians. Physicians were found, independent of specialty, to prescribe more frequently when they considered themselves the patient's primary care physician. This contrasts with earlier observations, in which no difference was found across physicians specialties, ${ }^{4}$ but is consistent with findings that family practitioners and other primary care physicians were higher prescribers of antibiotics for colds in children than pediatricians. ${ }^{9,10}$ This may suggest that physicians who are more familiar with their patients adopt a more subjective way of prescribing and are more influenced by the patient's expectations and requests, resulting in a lower compliance with clinical practice guidelines.

Disparities in training, continuous education, and access to relevant information might also explain these differences across physicians' groups. Recent training has been linked to reduced antibiotic prescription in 2 recent publications. Stone and colleagues showed that residents and interns, closer to their medical training, were less likely to prescribe antibiotics. ${ }^{8}$ Likewise, Mainous and colleagues ${ }^{9}$ found that for high prescribers of antibiotics, more years had passed since medical school. Although our findings might suggest that future educational campaigns and information tools should continue to target non-general internal medicine physicians and primary care physicians, a closer look at the predicted values for antibiotic prescribing across physician specialties reveals that there was no improvement in antibiotic prescription among general internal medicine physicians during the 3 years of observation, contrasting with a constant decrease in antibiotic prescription for general/family medicine physicians. Therefore, efforts should not only be targeted toward general/ family medicine physicians to sustain the favorable observed trend but should also be directed toward general internal medicine physicians.

Ethnicity was identified as another significant predictor. Hispanic patients seemed to be "protected" against antibiotic overuse, having 83\% lower chance than non-Hispanic patients of being prescribed an antibiotic for an illness. Ethnicity has not been systematically explored in previous studies, but our results contrast with those reported by Stone et $\mathrm{al}^{8}$, who did not identify ethnicity as a predictor for antibiotic prescription in a population of patients in emergency departments. Although there is no straightforward explanation for our findings, they are consistent with a significant increase in antibiotic prescriptions for cough observed in white/non-Hispanic patients in previous analyses of the NAMCS database. ${ }^{6}$ The use of a secondary database limits our ability to explore social and educational factors, beliefs about the efficacy of antibiotics, and severity of symptoms, all of which have been shown to influence antibiotic prescription and to have high variations across patients' populations. ${ }^{1-13}$ Because no information on patient socioeconomic background, education, or beliefs is collected in the NAMCS, a prospective collection of data would be more appropriate to address these issues.

In conclusion, an encouraging decrease in antibiotic prescription was observed in this study, but antibiotic overuse persists across physician specialties, necessitating better strategies to improve clinical practice. Although the dissemination of evidence-based clinical practice guidelines is one of the most commonly recognized tools used to reach 
this objective, the impact of the most recently published guidelines on the use of antibiotics for URIs $^{14-16}$ should be carefully monitored in the future to identify new predictors of antibiotic misuse and to develop more specific intervention programs. A better understanding of patients' expectations ${ }^{17}$ could help develop intervention programs in adult populations similar to those that had a positive impact among children. ${ }^{18,19}$

We thank E. Costich, D. Tornatore, R. Lopez, and N. Nadkarni for help in a first analysis of the data.

\section{References}

1. Dowell SF, Schwartz B. Resistant pneumococci: protecting patients through judicious use of antibiotics. Am Fam Physician 1997;55:1647-54.

2. Guillemot D, Carbon C, Balkau B, Geslin P, Lecoeur H, Vauzelle-Kervroedan F, et al. Low dosage and long treatment duration of beta-lactam: risk factors for carriage of penicillin-resistant Streptococcus pneumoniae. JAMA 1998;279:365-70.

3. Kaiser L, Lew D, Hirschel B, Auckenthaler R, Morabia A, Heald A, et al. Effects of antibiotic treatment in the subset of common-cold patients who have bacteria in nasopharyngeal secretions. Lancet 1996; 347:1507-10.

4. Gonzales R, Steiner JF, Sande MA. Antibiotic prescribing for adults with colds, upper respiratory tract infections, and bronchitis by ambulatory care physicians. JAMA 1997;278:901-4.

5. Tenney JB, White KL, Williamson JW. National Ambulatory medical care survey: background and methodology, United States, 1967-1972; a report on feasibility studies of methods developed for collecting national ambulatory medical care data from practicing office-based physicians in the United States. Vital and Health Statistics, series 2, no 61. DHEW publication no (HRA) 74-1335. Rockville (MD): National Center for Health Statistics; 1974.

6. Metlay JP, Stafford RS, Singer DE. National trends in the use of antibiotics by primary care physicians for adult patients with cough. Arch Intern Med 1998; 158:1813-8.

7. Gonzales R, Barrett PH, Crane LA, Steiner JF. Factors associated with antibiotic use for acute bronchitis. J Gen Intern Med 1998;13:541-8.

8. Stone S, Gonzales R, Maselli J, Lowenstein SR.
Antibiotic prescribing for patients with colds, upper respiratory tract infections, and bronchitis: a national study of hospital-based emergency departments. Ann Emerg Med 2000;36:353-5.

9. Mainous AG 3rd, Hueston WJ, Love MM. Antibiotics for colds in children: who are the high prescribers? Arch Pediatr Adolesc Med 1998;152: 349-52.

10. Nyquist AC, Gonzales R, Steiner JF, Sande MA. Antibiotic prescribing for children with colds, upper respiratory tract infections, and bronchitis. JAMA 1998;279:875-7.

11. Hong JS, Philbrick JT, Schorling JB. Treatment of upper respiratory infections: do patients really want antibiotics? Am J Med 1999;107:511-5.

12. Braun BL, Fowles JB. Characteristics and experiences of parents and adults who want antibiotics for cold symptoms. Arch Fam Med 2000;9:589-95.

13. Zoorob RJ, Larzelere MM, Malpani S, Zoorob R. Use and perceptions of antibiotics for upper respiratory infections among college students. J Fam Pract 2001;50:32-7.

14. Gonzales R, Bartlett JG, Besser RE, Hickner JM, Hoffman JR, Sande MA. Principles of appropriate antibiotic use for treatment of nonspecific upper respiratory tract infections in adults: background. Ann Intern Med 2001;134:490-4.

15. Snow V, Mottur-Pilson C, Gonzales R. Principles of appropriate antibiotic use for treatment of nonspecific upper respiratory tract infections in adults. Ann Intern Med 2001;134:487-9.

16. Gonzales R, Bartlett JG, Besser RE, Cooper RJ, Hickner JM, Hoffman JR, et al. Principles of appropriate antibiotic use for treatment of acute respiratory tract infections in adults: background, specific aims, and methods. Ann Intern Med 2001;134: 479-86.

17. Hamm RM, Hicks RJ, Bemben DA. Antibiotics and respiratory infections: are patients more satisfied when expectations are met? J Fam Pract 1996;43: 56-62.

18. Perz JF, Craig AS, Coffey CS, Jorgensen DM, Mitchel E, Hall S, et al. Changes in antibiotic prescribing for children after a community-wide campaign. JAMA 2002;287:3103-9.

19. Trepka MJ, Belongia EA, Chyou PH, Davis JP, Schwartz B. The effect of a community intervention trial on parental knowledge and awareness of antibiotic resistance and appropriate antibiotic use in children. Pediatrics 2001;107:E6. 